Advance Journal of Food Science and Technology 5(6): 732-737, 2013

DOI:10.19026/ajfst.5.3156

ISSN: 2042-4868; e-ISSN: 2042-4876

(C) 2013 Maxwell Scientific Publication Corp.

Submitted: January 31, $2013 \quad$ Accepted: February 25, 2013

Published: June 05, 2013

\title{
Research Article \\ Culture Conditions affect the Category and Production of Ubiquinones in a Recombinant Escherichia Coli with an Exogenous Decaprenyl Diphosphate Synthase
}

\author{
${ }^{1,2}$ Jiazhou Li, ${ }^{2}$ Yuanyuan Zhang, ${ }^{1}$ Mingquan Xie and ${ }^{1}$ Xiaochun Luo \\ ${ }^{1}$ School of Bioscience and Bioengineer, South China University of Technology, \\ Guangzhou 510640, China \\ ${ }^{2}$ Department of Food and Bioengineering, Guangdong Industry Technical College, \\ Guangzhou 510300, China
}

\begin{abstract}
Ubiquinones (UQ) are important electron transporters and play lot of important roles in most organisms. In different species, UQ was classified to be UQ-6, 7, 8, 9, 10 according to their polyprenyl side chain length. The side chain's length is determined by the enzyme named Poly-Prenyl diphosphate synthases (PPPS). Bacteria are usually reconstructed to producing UQ-10 used in human's food additive, medicine or cosmetics, such as using decaprenyl Diphosphate Synthase (DPS) gene from R. radiobacter to substitute E.coli's octaprenyl diphosphate synthase gene, just like E. coli BL21 ( $\triangle$ ispB::ddsA) used in this study. It is interesting that not only in these reconstructed bacteria, but in human-being, DPS can synthesize UQ-9 besides UQ-10. The mechanism of this phenomenon is still unknown. In this study, the effects of culture conditions, including the temperature, dissolved oxygen, $\mathrm{pH}$ and culture medium, on the DPS characteristics in E. coli BL21 ( $\triangle \mathrm{ispB}:: \mathrm{ddsA}$ ) were examined. Results show that temperature greatly affects the ratio of UQ-9/UQ-10, but not the total ubiquinone's production. Increasing dissolved oxygen and protein concentration in culture medium can promote total ubiquinone's production, but not the ratio of UQ-9/UQ-10. These results may give reference for UQ-10's industrial produce and the mechanism of these conditions' effect on DPS will be discussed.
\end{abstract}

Keywords: Decaprenyl diphosphate synthase, Escherichia coli, Rhizobium radiobacter, ubiquinone

\section{INTRODUCTION}

Ubiquinones (Coenzyme $\mathrm{Q}$, UQ or $\mathrm{CoQ}$ ) are essential components for organisms. Their basic function is to transfer electrons in respiratory chain from complex I/II to the cytochrome bcl complex in the inner membrane of eukaryote mitochondrion or the plasma membrane of prokaryote (Battino et al., 1990; Brandt and Trumpower, 1994; Lenaz and Genova, 2009). Additionally, ubiquinones have lots of other important functions as the following: UQ can function as the lipid-soluble antioxidants preventing the oxidation of DNA, proteins and lipids (Maroz et al., 2009); UQ regulates the cytosolic ratio of $\mathrm{NAD}+/ \mathrm{NADH}$ ratio involved in cell growth and differention (Gomezdiaz et al., 1997); UQ prevents the collapse of mitochondrial function by maintaining the integrity of mitochondrial membrane transition pore (Papucci et al., 2003); The oxidized UQ is an obligatory cofactor to assist uncoupling protein in the inner mitochondrial membrane to translocate protons (Echtay et al., 2001); UQ influences the expression of $\mathrm{NF} \kappa \mathrm{B} 1$-dependent genes involved in anti-inflammation (Schmelzer et al., 2007); CoQ can protect LDL from oxidation, which has anti-atherosclerotic properties (Thomas et al., 1996; Turunen et al., 2002); UQ can stimulate endothelial release of nitric oxide to counteract endothelial dysfunction (Hamilton et al., 2007); UQ mediates the disulfide bonds formation in bacterial and fungal proteins (Bader et al., 2000). In the past years, the research on UQ synthesis was intensively carried out and it is used as food additive, medicine or cosmetics universally (Clarke, 2000; Littarru and Tiano, 2007).

Ubiquinone consists of a redox active benzoquinone ring (4-hydroxybenzoic acid, 4-HB) attached to a polyprenyl side chain. After covalent bond formation between 4-HB and polypreny tail, the resulting 3-polyprenyl-4-hydroxybenzoate (HHB) undergoes several modifications such as hydroxylation, methylation and decarboxylation (Kawamukai, 2002). According to the length of poly-prenyl side chain, ubiquinones could be classified into UQ-6, UQ-7, UQ8, UQ-9 and UQ-10 and different UQ exists in different species. For example, UQ in mice is UQ-9, while in humans UQ-10 is the dominant UQ. In microorganisms, Saccharomyces cerevisiae UQ is UQ6, E. coli UQ is UQ-8. The different UQ species

\footnotetext{
Corresponding Author: Xiaochun Luo, School of Bioscience and Bioengineer, South China University of Technology, Guangzhou 510640, China

This work is licensed under a Creative Commons Attribution 4.0 International License (URL: http://creativecommons.org/licenses/by/4.0/).
} 
execute similar functions and could be artificially substituted with each other (Huang et al., 2011; Li et al., 2012).

The side chain is synthesized by poly-prenyl diphosphate synthases (PPPS), which belong to transprenytransferases family and catalyze chain elongation of Farnesyl Pyrophosphate (FPP) substrates via consecutive condensation reactions with Isopentenyl Pyrophosphate (IPP) to generate linear polymers with defined chain length (Sanchez et al., 2006). The FPP is synthesized by other poly-prenyl diphosphate synthase, namely farnesyl diphosphate synthase, from IPP and its isomer dimethaylallyl pyrophosphate (Yoon et al., 2006; Gu et al., 2011). The crystal structures of some PPPS had been reported (Guo et al., 2004; Chang et al., 2006; Sasaki et al., 2011). Results shows that two Asprich motifs, $\mathrm{DD}(\mathrm{XX}) \mathrm{nD}$ (X represents any amino acid and $n=2$ or 4) exist in these enzymes amino sequences, the first one responsible for FPP binding and the second for IPP (Kellogg and Poulter, 1997; Ogura and Koyama, 1998). After condensation, the hydrophobic tail of poly-prenyl chain extends into the cavity of the PPPS. The depth of cavity determines the length of chain. Tarshis et al. (1996) showed the side chains of residues corresponding to F112 and F113 of avian FPP synthase are very important for determining the ultimate length of poly-prenyl chain. By site directed mutagenesis, the avian FPP synthase was transformed into synthases capable of producing geranylgeranyl diphosphate (F112A), gernylfarnesyl diphosphate (F113S) and longer prenyl chains (F112A/F113S). By $\mathrm{X}$-ray analysis, the cavity of the F112A/F113S mutant increases in depth by $5.8 \AA$ compared with that for the wild-type enzyme. Guo et al. (2004) also proved the F132A mutant of octaprenyl diphosphate synthase from Thermotoga maritima can produce decaprenyl diphosphate.

The prenyl chains length are believed to be determined by PPPS, but it is interesting that the PPPS in some species can produce more than one prenyl chain with different length. For example, in human, the decaprenyl diphosphate synthase can synthesize UQ-9 besides UQ-10 (Aberg et al., 1992). In E. coli the octaprenyl diphosphate synthase can synthesize UQ-7 besides UQ-8. In our previous study when E. coli BL21 ( $\triangle$ ispB::ddsA) was constructed by substituting $E$. coli octaprenyl diphosphate synthase with decaprenyl Diphosphate Synthase (DPS) from R. radiobacter to produce UQ-10 (Li et al., 2012), we also found that $E$. coli BL21 ( $\triangle$ ispB::ddsA) can synthesize UQ-9 besides UQ-10. What factors affect the synthesis of different length UQ is still unknown.

In this study, to see if the culture conditions affect the production of different length UQ, the ratio of UQ-9 and UQ-10 from E. coli BL21 ( $\triangle$ ispB::ddsA) cultured under different temperatures, dissolved oxygen, $\mathrm{pH}$ and culture medium were tested. This study may give reference for promoting UQ-10 production in industry. The possible reason why these conditions affect the characteristics of DPS will also be discussed.

\section{MATERIALS AND METHODS}

Microbial used in this study was E. coli BL21 $(\triangle$ ispB::ddsA) constructed by our lab (Datsenko and Wanner, 2000). The DPS gene was cloned from R. radiobacter. Luria-Bertani (LB), tryptone and yeast extract were purchased from Oxoid LTD. Ubiquinone10 and -9 were purchased from Sigma Chemical Co. (Shanghai) used as standard samples. Ethanol, methanol and n-hexane were chromatographic grade purchased from Yongda chemical reagent Co. (Tianjin). All the other chemicals were analytic grade.

Methodology: Escherichia coli BL21 ( $\triangle$ ispB::ddsA) was pre-cultivated with LB medium in shake flask for overnight at $220 \mathrm{rpm}$ and $37^{\circ} \mathrm{C}$ to be used as seed culture. For exploring the temperature effect on characteristics of DPS in vivo, the temperature $24^{\circ} \mathrm{C}$, $27^{\circ} \mathrm{C}, 30^{\circ} \mathrm{C}, 33^{\circ} \mathrm{C}, 36^{\circ} \mathrm{C}$ and $39^{\circ} \mathrm{C}$ were chosen for test. Under each temperature, triple $50 \mathrm{ml} \mathrm{LB}$ broth (pH 7.2) in $250 \mathrm{ml}$ flasks were inoculated with $2 \mathrm{ml}$ seed culture and cultivated at $220 \mathrm{rpm}$ for $12 \mathrm{~h}$.

For exploring the DO effect on characteristics of DPS in vivo, different shaking speed of shaker was chosen. Under 0, 40, 80, 120, 160, 200 and $240 \mathrm{rpm}$, triple $50 \mathrm{ml} \mathrm{LB}$ broth $(\mathrm{pH} 7.2)$ in $250 \mathrm{ml}$ flasks were inoculated with $2 \mathrm{ml}$ seed culture and incubated for 12 $\mathrm{h}$ at $37^{\circ} \mathrm{C}$ respectively.

With the initial $\mathrm{pH}$ of $6.0,6.5,7.0$ and $7.5,2 \mathrm{ml}$ seed culture was transferred into the $50 \mathrm{ml} \mathrm{LB}$ medium in $250 \mathrm{ml}$ flask respectively. The flasks were shaked in a rotary shaker at $220 \mathrm{rpm}$ and $37^{\circ} \mathrm{C}$ for $12 \mathrm{~h}$.

Peptone was chosen as limited protein nutrition in LB medium. Changing the peptone concentration, nutrition condition effect on characteristics of DPS was studied. As preceding methods, experiments were carried out under different peptone concentration as $0.25,0.50,0.75,1.00$ and $1.25 \%$ respectively. Each was repeated three times.

Analysis Methods: Cell density of fermentation broth was measured with optical density at $600 \mathrm{~nm}$ and converted to dry cell mass $(\mathrm{g} / \mathrm{L})$ according to a preprepared standard curve. Cells were collected from 50 $\mathrm{ml}$ fermentation broth by centrifuging at $3214 \mathrm{~g}$ for 8 min and stored at $-20^{\circ} \mathrm{C}$ for UQ extraction. Cells pellet was suspended in $5 \mathrm{ml} \mathrm{n}$-hexane and sonicated for 15 min. Cells debris was suspended in $5 \mathrm{ml} n$-hexane and sonicated for $15 \mathrm{~min}$. Cells debris was collected by centrifuge and extracted with n-hexane one more time. These tow supernatants were mixed and vacuum evaporation was used to remove the solvent, $\mathrm{n}$-hexane. The dry ubiquinone sample was resolved in $1 \mathrm{~mL}$ chromatographic ethanol for further test.

CoQ was detected according to the methods described before (Yen and Shih, 2009). High Performance Liquid Chromatograph (HPLC) was carried out with Prominence LC-20A (SHIMADZU, 
Janpan) equipped with shodex C18M 4E (250 mm $\times 4.6$ $\mathrm{mm})$ column. Mixed liquid of ethanol and methanol (7:3) was used as mobile phase at a flow rate of 1 $\mathrm{ml} / \mathrm{min}$. UV detector at $275 \mathrm{~nm}$ was used.

\section{RESULTS AND DISCUSSION}

The E. coli BL21 ( $\triangle$ ispB::ddsA) was cultured under different temperatures and the UQ was tested by HPLC. The results were shown in Fig. 1.

In this figure, we can see when the temperature was less than $30^{\circ} \mathrm{C}$, only UQ- 10 was detected. While when temperature was above $30^{\circ} \mathrm{C}$, the UQ-9 began to be synthesized and the ratio of UQ-9 to UQ-10 increased with the rise of temperature. At $39^{\circ} \mathrm{C}$, the concentration of UQ-9 is $0.21 \mathrm{mmol} / \mathrm{g} \mathrm{DCM}$ (mmol per gram dry cell mass), higher than the concentration of UQ-10 $(0.12 \mathrm{mmol} / \mathrm{g}$ DCM $)$. While the total UQ at different temperature showed no great difference and sustained about $0.33 \mathrm{mmol} / \mathrm{g}$ DCM. These results show the temperature may not affect the catalytic efficiency of DPS, while affect the dissociation of product from the enzyme. When the temperature above $30^{\circ} \mathrm{C}$, as the synthesis proceeded to be UQ-9, some of them dissociated from DPS enzyme before the last IPP polymerized.

Naturally, there are three kinds of polyprenyl diphosphate synthases, namely cis-, trans-, cis- and trans- mixed (Kera et al., 2012). Cis- forms appear intensively in microorganisms and plants and produce long chain cis-polyprenyl diphosphate with eleven isopentenyl units, such as undecaprenyl diphosphate synthase for peptidoglycan biosynthesis (Teng and Liang, 2012), to thousands units, such as natural rubber (Takahashi et al., 2012). Trans- forms are responsible for biosynthesis of relatively short polyprenyl chains, such as UQ and lycopene (Bhataya et al., 2009). The physiological functions of cis- and trans- mixed are not elucidated until now. About the length determination mechanism of polyprenyl chain, all researches were based on the structures of the synthases. About the trans-form, it was been reported that the length of polyprenyl chain of UQ is determined by the depth of the cavity formed by two DPS homo-dimmer subunit. The bulge side chains of two Phe in this enzyme cavity stop the elongation of the polyprenyl tail and then the products dissociated from the enzyme. But this can not be used to explain why the same synthase can produce different length UQ in vivo, such as that in human tissues; UQ-9 occupies $2-5 \%$ of total ubiquinone. Then the environment conditions may be the most suspect factors.

In our study, we found the E. coli BL21 ( $\triangle$ ispB::ddsA), which octaprenyl diphosphate synthase gene was replaced by ddsA from R. radiobacter, could produce UQ-9 besides UQ-10 under temperature

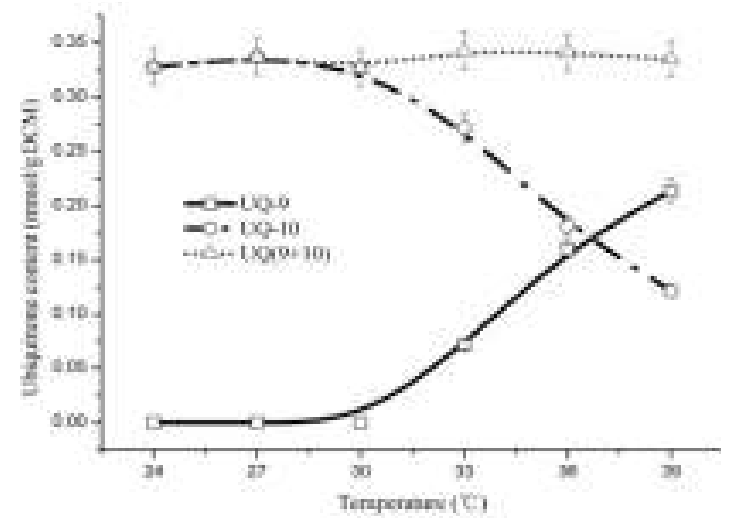

Fig. 1: Temperature effect on characteristics of DPS from $R$. radiobacter in $E$. coli

over $30^{\circ} \mathrm{C}$. Schmelzer et al. (2007) had reported that CoQ10 affects NFKB-related genes' expression (such as IL5, thrombin, vitronectin receptor and cAMP receptor protein) and CoQ10-inducible genes play an important role in inflammatory response. There may be an implication that human's temperature rise in inflammation situation affects DPS' UQ synthesis and that the ratio change of UQ-9/UQ-10 affects inflammatory response. The ratio of UQ-9/UQ-10 may be looked as a sign of inflammation of body. All proteins' functions were connected with them structures tightly. We can conclude that the high temperature would affected the structure of enzyme active center and promote the UQ-9'dissociation. But no such obvious phenomenon was reported about DPS from other species, such as Agrobacterium tumefaciens (Ha et al., 2007), Rhodobacter sphaeroides (Zahiri et al., 2006), Gluconobacter suboxydans (Okada et al., 1998) and so on. These mean DPS from different species have different stabilities. DPS from R. radiobacter is more unstable than others and more sensitive to temperature. Our result also indicated that temperature is important factor in bacteria producing UQ-10 in industry. From the stability of molar sum of ubiquinones content, it can be concluded that the feedback regulation of ubiquinone synthesis is irrelevant with the kind of ubiquinones.

According to the curve in Fig. 1, the following equations can be got to show the relationship between the concentration of UQ9 (y, mmol/g DCM), UQ10 (x, $\mathrm{mmol} / \mathrm{g} \mathrm{DCM})$ and the temperature $\left(\mathrm{T},{ }^{\circ} \mathrm{C}\right)$ :

$$
\left\{\begin{array}{l}
y=0.0243 T-0.7273 \\
x=-0.0236 T+1.0386 \\
y+x=0.335113
\end{array}\right.
$$

Ubiquinone is important in electron transport of cell respiration. DO concentration's effect on E. coli BL21 ( $\triangle$ ispB:::ddsA) producing ubiquinones at $37^{\circ} \mathrm{C}$ was shown in Fig. 2. 


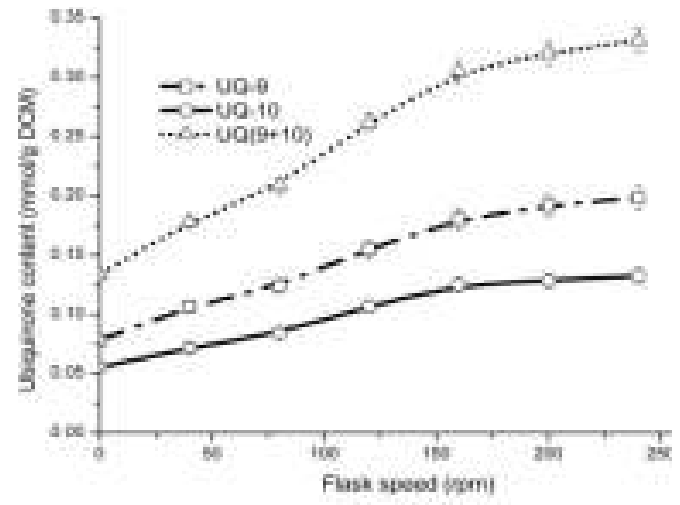

Fig. 2: DO concentration effect on characteristics of DPS from $R$. radiobacter in $E$. coli

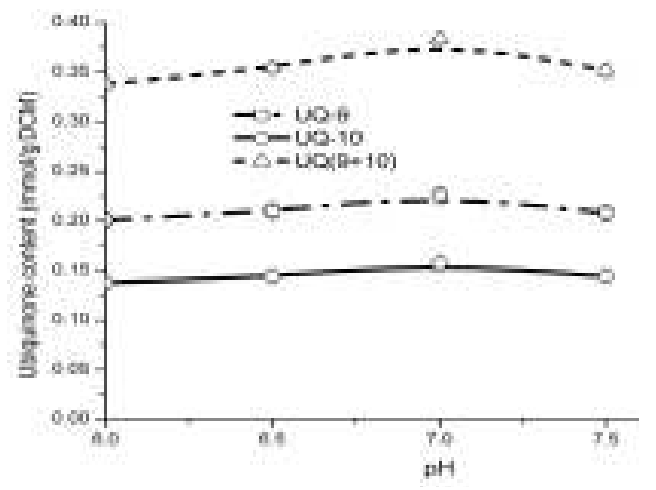

Fig. 3: $\mathrm{pH}$ effect on characteristics of DPS from $R$. radiobacter in E. coli

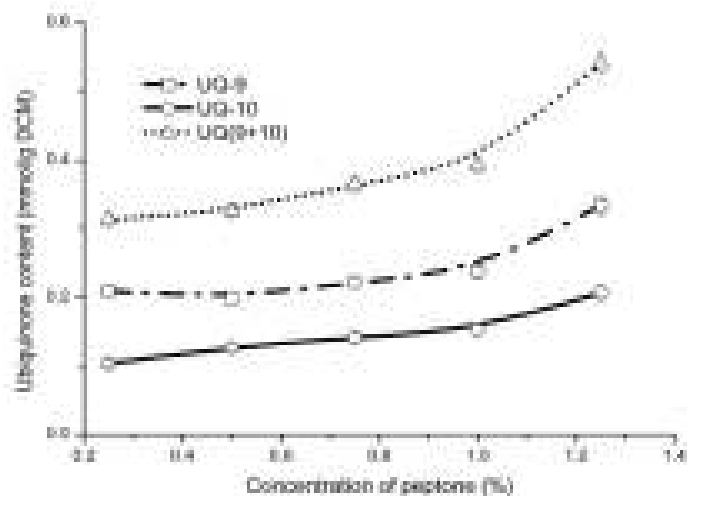

Fig. 4: Concentration of peptone effect on characteristics of DPS from $R$. radiobacter in $E$. coli

We can see with the increase of DO concentration, both the production of UQ-9 and UQ-10 in E. coli BL21 ( $\triangle$ ispB::ddsA) increase. These results indicated increasing DO concentration in these bacteria promotes the bacteria's respiration and the ubiquinone production increases. The production of total ubiquinone was promoted in E. coli BL21 ( $\triangle$ ispB::ddsA) with OD concentration increasing, while the ratio of UQ-9/UQ-
10 remain steadily, which indicates OD concentration does not affect the DPS catalysis properties.

Yoshida et al. (1998) reported R. sphaeroides could increase yield of ubiquinone 10 under limited supply of air, but R. radiobacter is quite the opposite. It has been reported when DO concentration increases in a certain range, the CoQ10 production decreases in $\mathrm{R}$. radiobacter just like our results (Wu et al., 2003). Both R. radiobacter and E. coli are facultative anaerobe and have two kinds of respiratory pathway. In strictly aerobic bacterium, it compensates oxygen lack by adding the respiratory chain and leads to ubiquinone increasing. In facultative anaerobes, it can compensate the oxygen lack by anaerobic respiration without ubiquinone increasing. On the contrary, ubiquinone will decrease for aerobic respiration demand reduction.

The effects of $\mathrm{pH}$ and peptone concentration on the production of UQ-9 and UQ-10 at $37^{\circ} \mathrm{C}$ were respectively shown in Fig. 3 and 4. Results showed both $\mathrm{pH}$ and peptone concentration did not apparently affect the ratio of UQ-9/UQ-10. $\mathrm{pH}$ is an important factor for enzyme activity. In this study, culture medium's $\mathrm{pH}$ may not affect the $\mathrm{pH}$ inner cell. So the $\mathrm{pH}$ of culture medium seems no affect to DPS enzyme properties. Increasing the concentration of peptone can increase both UQ-9 and UQ-10 production (Fig. 4), but this factor may not affect DPS enzyme properties as $\mathrm{pH}$ or they have no direct relationship with intracellular enzymes' activity or stability.

\section{CONCLUSION}

In summary, our results show that higher temperature above $30^{\circ} \mathrm{C}$ may cause the earlier release of nonaprenyl diphosphate from DPS and reduce the production of decaprenyl diphosphate. Increasing dissolved oxygen and protein concentration in culture medium can promote total ubiquinone's production, but not the ratio of UQ-9/UQ-10. These results indicate the fermentation of UQ-10 industrial produce ought to be control at an appropriate temperature to yield higher UQ-10 ratio and increasing DO and protein supplement may help the higher yield of total ubiquinones.

\section{ACKNOWLEDGMENT}

The authors thank the natural science foundation of Guangdong Province (7004113) and the Projects of Science and Technology of Guangdong Province (2011B010500018).

\section{REFERENCES}

Aberg, F., E.L. Appelkvist, G. Dallner and L. Ernster, 1992. Distribution and redox state of ubiquinones in rat and human tissues. Arch. Biochem. Biophys., 295: 230-234. 
Bader, M.W., T. Xie, C.A. Yu and J.C. Bardwell, 2000. Disulfide bonds are generated by quinone reduction. J. Biol. Chem., 275: 26082-26088.

Battino, M., E. Ferri, A. Gorini, R.F. Villa, J.F. Rodriguez Huertas, P. Fiorella, M.L. Genova, G. Lenaz and M. Marchetti, 1990. Natural distribution and occurrence of coenzyme Q homologues. Membr. Biochem., 9: 179-190.

Bhataya, A., C. Schmidt-Dannert and P.C. Lee, 2009. Metabolic engineering of Pichia pastoris X-33 for lycopene production. Process. Biochem., 44: 1095-1102.

Brandt, U. and B. Trumpower, 1994. The protonmotive Q cycle in mitochondria and bacteria. Crit. Rev. Biochem. Mol. Biol., 29: 165-197.

Chang, H.T., R.T. Guo, T.P. Ko, A.H. Wang and P.H. Liang, 2006. Crystal of structure-III geranylgeranyl pyrophosphate synthase from Saccharomyces cerevisiae and the mechanism of product chain length determination. J. Biol. Chem., 281: 14991-15000.

Clarke, C.F., 2000. New advance in coenzyme Q biosynthesis. Protoplasma, 213: 134-147.

Datsenko, K.A. and B.L. Wanner, 2000. One-step inactivation of chromosomal genes in Escherichia coli K-12 using PCR products. Proc. Natl. Acad. Sci. USA, 97: 6640-6645.

Echtay, K.S., E. Winkler, K. Frischmuth and M. Klingenberg, 2001. Uncoupling proteins 2 and 3 are highly active $\mathrm{H}(+)$ transporters and highly nucleotide sensitive when activated by coenzyme Q (ubiquinone). Proc. Natl. Acad. Sci. USA, 98: 1416-1421.

Gomezdiaz, C., J.C. RodriguezAguilera, M.P. Barroso, J.M. Villalba, F. Navarro, F.L. Crane and P. Navas, 1997. Antioxidant ascorbate is stabilized by NADH-coenzyme Q(10) reductase in the plasma membrane. J. Bioenerg. Biomembr., 29: 251-257.

Gu, W., Q.N. Wu, J.G. Chao, B.L. Xi, L. Li and X.Y. Shen, 2011. Molecular cloning of farnesyl pyrophosphate synthase from Alisma orientale (Sam.) Juzep. and its distribution pattern and bioinformatics analysis. Acta Pharmacol. Sin., 46: 605-612.

Guo, R.T., C.J. Kuo, C.C. Chou, T.P. Ko, H.L. Shr, P.H. Liang and A.H. Wang, 2004. Crystal structure of octaprenyl pyrophosphate synthase from hyperthermophilic Thermotoga maritima and mechanism of product chain length determination. J. Biol. Chem., 279: 4903-4912.

Ha, S.J., S.Y. Kim, J.H. Seo, H.J. Moon, K.M. Lee and J.K. Lee, 2007. Controlling the sucrose concentration increases coenzyme Q10 production in fed-batch culture of Agrobacterium tumefaciens. Appl. Microbiol. Biotechnol., 76: 109-116.
Hamilton, S.J., G.T. Chew and G.F. Watts, 2007. Therapeutic regulation of endothelial dysfunction in type 2 diabetes mellitus. Diab. Vasc. Dis. Res., 4: 89-102.

Huang, M.T., Y. Wang, J.Z. Liu and Z.W. Mao, 2011. Multiple strategies for metabolic engineering of Escherichia coli for efficient production of coenzyme Q(10). Chinese J. Chem. Eng., 19: 316-326.

Kawamukai, M., 2002. Biosynthesis, bioproduction and novel roles of ubiquinone. J. Biosci. Bioeng., 94: 511-517.

Kellogg, B.A. and C.D. Poulter, 1997. Chain elongation in the isoprenoid biosynthetic pathway. Curr. Opin. Chem. Biol., 1: 570-578.

Kera, K., S. Takahashi, T. Sutoh, T. Koyama and T. Nakayama, 2012. Identification and characterization of a cis, trans-mixed heptaprenyl diphosphate synthase from Arabidopsis thaliana. FEBS J., 279: 3813-3827.

Lenaz, G. and M.L. Genova, 2009. Mobility and function of coenzyme $Q$ (ubiquinone) in the mitochondrial respiratory chain. Biochim. Biophys. Acta, 1787: 563-573.

Li, J.Z., M.Q. Xie, A.X. Li and X.C. Luo, 2012. Construction of engineering for Escherichia coli production of coenzyme Q10. J. South China Univ. Technol. (Natural Sci. Edn.), 5: 90-95.

Littarru, G.P. and L. Tiano, 2007. Bioenergetic and antioxidant properties of coenzyme Q10: Recent developments. Mol. Biotechnol., 37: 31-37.

Maroz, A., R.F. Anderson, R.A. Smith and M.P. Murphy, 2009. Reactivity of ubiquinone and ubiquinol with superoxide and the hydroperoxyl radical: Implications for in vivo antioxidant activity. Free Radic. Biol. Med., 46: 105-109.

Ogura, K. and T. Koyama, 1998. Enzymatic aspects of isoprenoid chain elongation. Chem. Rev., 98: 1263-1276.

Okada, K., T. Kainou, K. Tanaka, T. Nakagawa, H. Matsuda and M. Kawamukai, 1998. Molecular cloning and mutational analysis of the $d d s A$ gene encoding decaprenyl diphosphate synthase from Gluconobacter suboxydans. Eur. J. Biochem., 255: 52-59.

Papucci, L., N. Schiavone, E. Witort, M. Donnini, A. Lapucci, A. Tempestini, L. Formigli, S. ZecchiOrlandini, G. Orlandini, G. Carella, R. Brancato and S. Capaccioli, 2003. Coenzyme q10 prevents apoptosis by inhibiting mitochondrial depolarization independently of its free radical scavenging property. J. Biol. Chem., 278: 2822028228.

Sanchez, V.M., A. Crespo, J.S. Gutkind and A.G. Turjanski, 2006. Investigation of the catalytic mechanism of farnesyl pyrophosphate synthase by computer simulation. J. Phys. Chem. B., 110: 18052-18057. 
Sasaki, D., M. Fujihashi, N. Okuyama, Y. Kobayashi, M. Noike, T. Koyama and K. Miki, 2011. Crystal structure of heterodimeric hexaprenyl diphosphate synthase from Micrococcus luteus B-P 26 reveals that the small subunit is directly involved in the product chain length regulation. J. Biol. Chem., 286: 3729-3740.

Schmelzer, C., I. Lindner, C. Vock, K. Fujii and F. Doring, 2007. Functional connections and pathways of coenzyme Q10-inducible genes: An in-silico study. IUBMB Life, 59: 628-633.

Takahashi, S., H.J. Lee, S. Yamashita and T. Koyama, 2012. Characterization of cis-prenyltransferases from the rubber producing plant Hevea brasiliensis heterologously expressed in yeast and plant cells. Plant Biotech., 29: 411-417.

Tarshis, L.C., P.J. Proteau, B.A. Kellogg, J.C. Sacchettini and C.D. Poulter, 1996. Regulation of product chain length by isoprenyl diphosphate synthases. Proc. Natl. Acad. Sci. USA, 93: 15018-15023.

Teng, K.H. and P.H. Liang, 2012. Structure, mechanisms and inhibitors of undecaprenyl diphosphate synthase: A cis-prenyltransferase for bacterial peptidoglycan biosynthesis. Bioorg. Chem., 43: 51-57.

Thomas, S.R., J. Neuzil and R. Stocker, 1996. Cosupplementation with coenzyme Q prevents the prooxidant effect of alpha-tocopherol and increases the resistance of LDL to transition metal-dependent oxidation initiation. Arterioscl. Throm. Vas., 16: 687-696.
Turunen, M., L. Wehlin, M. Sjoberg, J. Lundahl, G. Dallner, K. Brismar and P.J. Sindelar, 2002. Beta2Integrin and lipid modifications indicate a nonantioxidant mechanism for the anti-atherogenic effect of dietary coenzyme Q10. Biochem. Biophys. Res. Commun., 296: 255-260.

Wu, Z.F., G.C. Du and J. Chen, 2003. Effects of dissolved oxygen concentration and DO-stat feeding strategy on CoQ10 production with Rhizobium radiobacter. World J. Microb. Biot., 19: 925-928.

Yen, H.W. and T.Y. Shih, 2009. Coenzyme Q10 production by rhodobacter sphaerroides in stirred tank and in airlift bioreactor. Bioproc. Biosyst. Eng., 32: 711-716.

Yoon, S.H., Y.M. Lee, J.E. Kim, S.H. Lee, J.H. Lee, J.Y. Kim, K.H. Jung, Y.C. Shin and J.D. Keasling, 2006. Enhanced lycopene production in Escherichia coli engineered to synthesize isopentenyl diphosphate and dimethylallyl diphosphate from mevalonate. Biotechnol. Bioeng., 94: 1025-1032.

Yoshida, H., Y. Kotani, K. Ochiai and K. Araki, 1998. Production of ubiquinone-10 using bacteria. J. Gen. Appl. Microbio., 44: 19-26.

Zahiri, H.S., K.A. Noghabi and Y.C. Shin, 2006. Biochemical characterization of the decaprenyl diphosphate synthase of Rhodobacter sphaeroides for coenzyme Q10 production. Appl. Microbiol. Biotechnol., 73: 796-806. 